\title{
Experimental ocular toxocariasis: a mouse model
}

\author{
S. Y. A. GHAFOOR, ${ }^{1}$ H. V. SMITH,${ }^{2}$ W. R. LEE, ${ }^{1}$ R. QUINN,${ }^{3}$ \\ AND R. W. A. GIRDWOOD ${ }^{2}$ \\ From the ${ }^{1}$ Tennent Institute of Ophthalmology, Western Infirmary, Glasgow; \\ the ${ }^{2}$ Bacteriology Department, Stobhill Hospital, Glasgow; and the ${ }^{3}$ Zoology Department, \\ University of Glasgow, Glasgow
}

SUMmaRY Male mice, strain C57 black, were infected with Toxocara canis by a single intragastric dose of 1500 infective eggs. The eyes were studied at sequential time periods after infection (6 to 63 days) by conventional microscopic techniques, and the histological characteristics of the inflammatory response were recorded. In the majority of animals the disease was unilateral. Twenty-six larvae were found in the retina, in the retinal vessels, and in the subretinal space in 20 eyes, while in 29 eyes there were inflammatory changes which were not related to the presence of intact or fragmented larval forms. The inflammatory reaction began as a polymorphonuclear response but after day 13 became a granulomatous reaction. This suggests that the inflammatory phenomenon may be propagated by the secreted surface antigens in the absence of the living or dead larvae.

Children and adults ${ }^{12}$ become infected by the accidental ingestion of dog faecal material containing Toxocara canis ova. The clinical manifestations of ocular toxocariasis are solitary and usually occur in older children (average 7.5 years) without the generalised eosinophilia, hyperglobulinaemia, and hepatomegaly characteristic of visceral larva migrans (VLM). ${ }^{3}$ Lesions occur unilaterally as a solitary retinal granuloma in the posterior pole in proximity to the disc and macula and/or as chronic endophthalmitis with retinal detachment. ${ }^{4}$ Wilder $^{5}$ found nematode larvae in 24 cases of pseudogliomata, and Nichols ${ }^{6}$ identified these larvae as Toxocara canis. In such studies eyes were usually enucleated because of the difficulty of excluding retinoblastoma.

Although ocular toxocariasis is an uncommon form of uveitis and retinitis in the routine uveitis clinic, ${ }^{7}$ treatment of the majority of cases in which there is an exudative retinal detachment is unrewarding. Subtotal pars plana vitrectomy ${ }^{8}$ has been added recently to the current therapeutic measures, which included topical and systemic corticosteroids, anthelminthic therapy, and laser photocoagulation. The pathogenic mechanisms which lead to retinal damage are poorly understood, notably the immunopathological interreactions between the host and the parasite, so that there is a dearth of information on which to base

Correspondence to S. Y. A. Ghafoor, FRCS, Tennent Institute of Ophthalmology, Western Infirmary, Glasgow G11 6NT. therapy. It is therefore of value to develop cheap and reliable animal models with a low degree of mortality and a high degree of morbidity in order to apply the rapidly developing techniques of immunohistochemistry to the study of nematode-induced inflammatory disease. To this end we present our findings in a preliminary study of ocular toxocariasis in the mouse. To our knowledge there has not been a detailed histological study over an extended period of the effects of toxocariasis on the pigmented eye of a laboratory animal.

\section{Materials and methods}

Animals. Three uninfected 10-week-old mice (C57 black strain) were used as controls to determine the normal ocular histology in this strain. Twenty-seven male C57 black mice, 10 weeks old, were infected with a single dose of 1500 infective eggs by intragastric intubation on day 0 , and 3 animals were chosen randomly and killed by cervical dislocation under anaesthesia on days $6,13,20,32,34,41,50,56$, and 63 after infection.

Isolation of infective eggs. Toxocara canis eggs were recovered from the uteri of adult female worms obtained from the gastrointestinal tracts of dogs at autopsy. The worms were washed and stored in $4 \%$ formalin at room temperature $\left(21^{\circ} \mathrm{C}\right)$ for 4 weeks. Following embryonation, which occurred while the adult worm was immersed in formalin, and develop- 


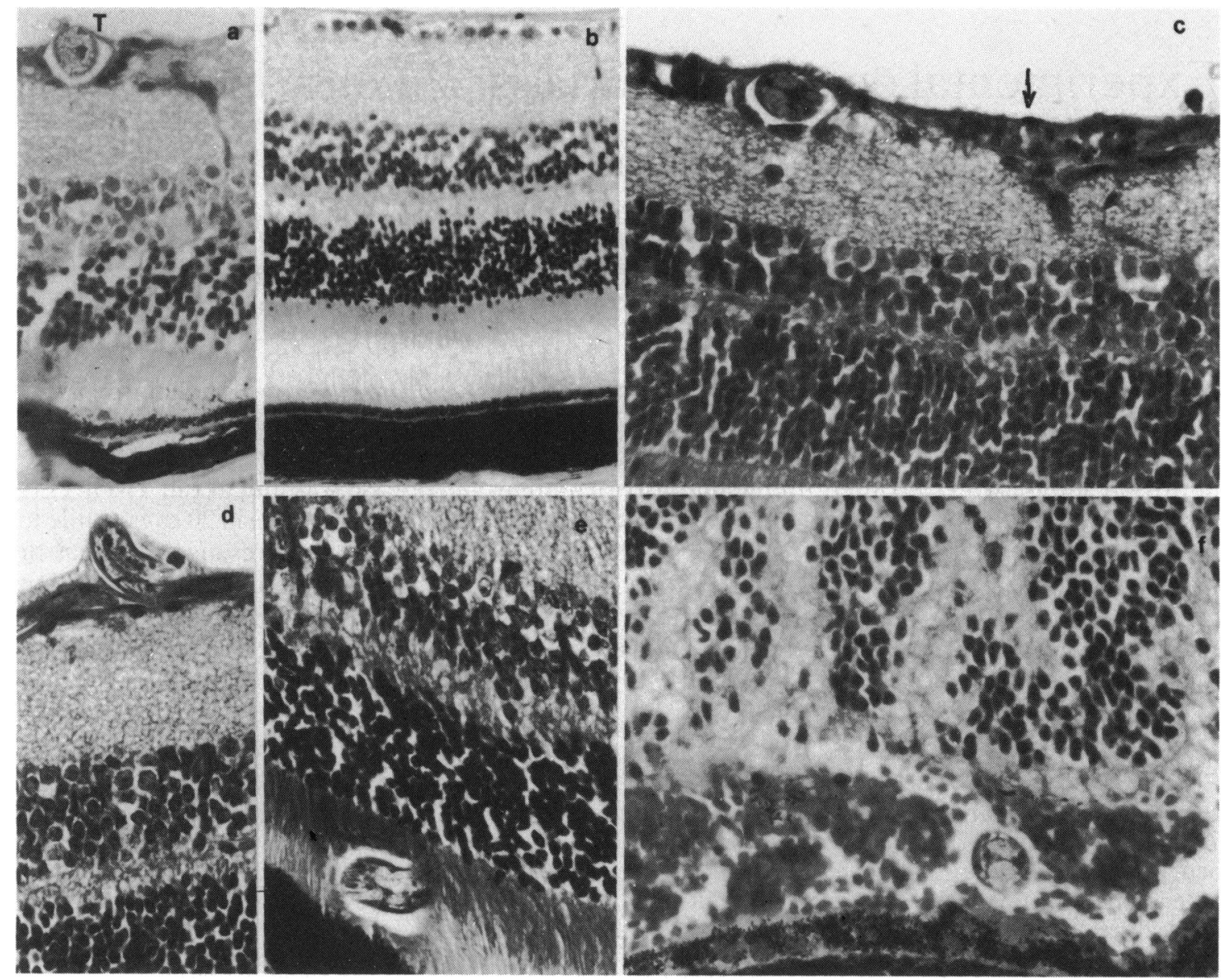

Fig. 1 Illustrations of Toxocara canis larva in the retina (paraffin sections stained $H$ and $E$ ). a: Toxocara canis larva in lumen of retinal capillary at day $13(T)(\times 630)$. b: Normal retina of a control mouse $(H$ and $E, \times 400)$. c: Toxocara canis larva lying in superficial retina; the adjacent vessel $(\downarrow)$ shows a vasculitis $(\times 400)$. d: Larva within a retinal capillary and the vitreous at day $56(\times 630)$. e: Larva in outer segments at day $6(\times 630)$. f: Larva within a subretinal haemorrhage at day $34(\times 630)$.

ment to the second larval stage $\left(\mathrm{L}_{2}\right)$ within the eggshell, the infective eggs were dissected from the uteri and washed extensively in phosphate buffered saline (PBS) $\mathrm{pH} 7 \cdot 1$, prior to use.

Pathological examination. The eyes and orbital tissues were removed immediately after death and were fixed in $2.5 \%$ gluteraldehyde in $0.4 \mathrm{M}$ cacodylate buffer, $\mathrm{pH} 7 \cdot 2$, at $4^{\circ} \mathrm{C}$. Each eye was opened and the lens was removed. The retina was examined for lesions under a dissecting microscope at appropriate magnification.

Histological examination. Specimens for light microscopy were embedded in paraffin, and serial sections $5 \mu \mathrm{m}$ in thickness were taken in the sagittal plane of the eye and stained with haematoxylin and eosin. Selected sections were stained with carbol chromotrope to demonstrate eosinophils. To assess the viability of $\mathrm{L}_{2}$ Toxocara canis larvae one sample from a bisected eye from each group of 3 mice was retained for transmission electron microscopy (TEM). These samples were rinsed in cacodylate buffer $(0 \cdot 2 \mathrm{M})$, postfixed in osmium tetroxide $(2 \%)$, dehydrated in graded alcohols, and embedded in Araldite. Semithin sections were taken and stained with toluidine blue. For the study of the preservation of the larval forms ultrathin sections were stained with uranyl acetate and lead citrate and examined in a Phillips 301 electron microscope.

\section{Results}

Macroscopic and microscopic examination of the control material revealed the normal architecture of the pigmented mouse eye (Fig. 1b). 


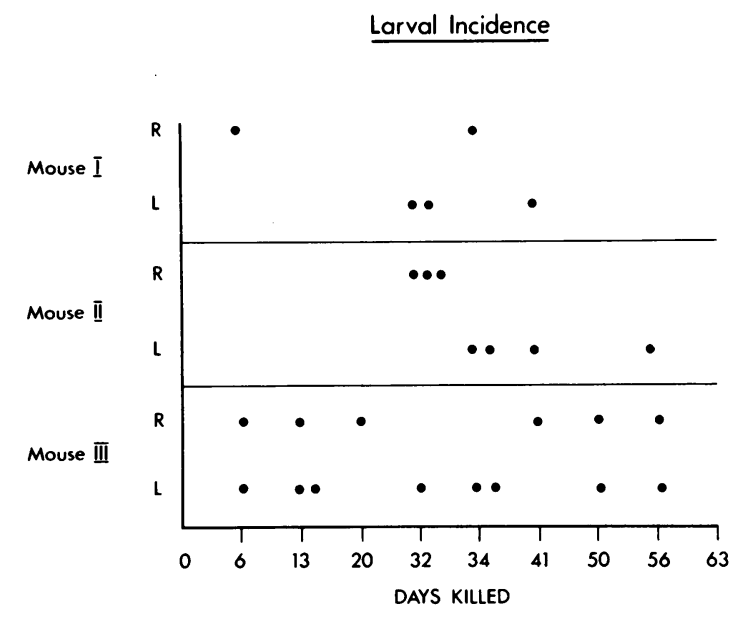

Fig. 2 Larval incidence in eyes of infected mice. $R$ is the right eye, $L$ is the left eye. I, II, and III denote a set of 3 randomly chosen mice killed on the days noted in the horizontal axis.

Macroscopic examination of the 54 eyes from 27 animals, which had been infected with Toxocara canis larvae revealed grey fluffy masses in the peripheral retina in 5 eyes only. Twenty-six larvae were found in serial sections from the experimental eyes (Table 1) and were recognised at each time interval throughout the experimental period (Fig. 2). The location of the larvae was mainly at the posterior

Table 1 Distribution of larvae in 54 eyes

\begin{tabular}{lr}
\hline \multicolumn{3}{l}{ Distribution of larvae } & \\
\hline Posterior pole & 18 \\
Peripheral retina & 5 \\
Vitreous & 1 \\
Retinal capillary & 2 \\
Total & 26 \\
\hline
\end{tabular}

pole (Table 1), but 5 were in the peripheral retina and one was present in the vitreous. On 2 occasions larvae were identified within the lumen of retinal capillaries (Fig. 1a, d), while the majority appeared to be lying within the retinal substance or were in the subretinal space (Fig. 1e, f).

The pathological changes which occurred in the eyes of the infected animals are summarised in Table 2 . Evidence of occlusive retinal disease was obtained most frequently in the early stages of infection (days 6 and 13), when superficial and deep haemorrhages were observed; the latter extended in some cases into the subretinal space (Fig. 1f). Microinfarcts were found commonly throughout the experimental period (Fig. 3a, Table 2). Haemorrhagic pseudocysts were found in the retina and the vitreous (Fig. 3b). Retinal vasculitis (Fig. 1c) was more frequently found in the later stages of the experiment.

It is noteworthy that on only one occasion was there evidence of an inflammatory reaction in the presence of a larval form (Fig. 3), and it was rarely possible to identify structures which could have been interpreted as dead or necrotic larval forms. Ultrastructural examination of the larvae showed the organism to have normal cell walls and organelles (Fig. 4).

Nongranulomatous inflammatory infiltrates, consisting of lymphocytes and plasma cells were found in the anterior uvea and the pars plana (Fig. 3d, e). This type of cellular infiltrate succeeded a polymorphonuclear leucocyte infiltrate which occurred in the first 13 days. The vitreous was commonly involved in the inflammatory process, but the infiltrates were mainly lymphocytic and haemorrhagic, and the macrophages within the vitreous body contained melanin and red cell degradation products (Fig. 3d, e, f). One circumscribed abscess was found in the vitreous adjacent to an intact larva (Fig. 3c).

A granulomatous response was rare and, apart

Table 2 The incidence of histopathological features in experimental toxocariasis. The numbers in each column refer to the number of eyes affected: 6 eyes were examined at each specified time period

\begin{tabular}{|c|c|c|c|c|c|c|c|c|c|c|c|}
\hline Lesion & Day & 6 & 13 & 20 & 32 & 34 & 41 & 50 & 56 & 63 & Total \\
\hline Iridocyclitis & & 0 & 0 & 5 & 5 & 1 & 2 & 3 & 1 & 3 & 20 \\
\hline Pars planitis & & 0 & 2 & 6 & 4 & 3 & 1 & 5 & 2 & 6 & 29 \\
\hline Vitreal haemorrhage & & 3 & 3 & 2 & 1 & 4 & 4 & 1 & 1 & 9 & 28 \\
\hline Vitreal infiltrate & & 3 & 2 & 5 & 4 & 4 & 4 & 6 & 3 & 6 & 37 \\
\hline Optic nerve papillitis & & 0 & 0 & 1 & 1 & 0 & 0 & 1 & 2 & 2 & 7 \\
\hline Retinal superficial haemorrhage & & 4 & 0 & 1 & 1 & 1 & 0 & 1 & 1 & 0 & 9 \\
\hline Retinal deep haemorrhage & & 3 & 0 & 0 & 1 & 0 & 1 & 1 & 0 & 0 & 6 \\
\hline Subretinal haemorrhage & & 4 & 1 & 1 & 1 & 0 & 1 & 0 & 0 & 0 & 8 \\
\hline Microinfarcts & & 3 & 4 & 1 & 4 & 3 & 3 & 3 & 4 & 2 & 27 \\
\hline Vasculitis & & 1 & 3 & 1 & 3 & 1 & 2 & 2 & 1 & 1 & 15 \\
\hline Subretinal infiltrate & & 0 & 0 & 0 & 4 & 1 & 2 & 2 & 2 & 0 & 11 \\
\hline Retinal granuloma & & 0 & 3 & 3 & 0 & 0 & 3 & 1 & 0 & 0 & 10 \\
\hline Choroid & & 0 & 0 & 0 & 0 & 0 & 0 & 0 & 0 & 0 & 0 \\
\hline Orbit & & 0 & 0 & 0 & 0 & 1 & 0 & 0 & 0 & 0 & 1 \\
\hline
\end{tabular}



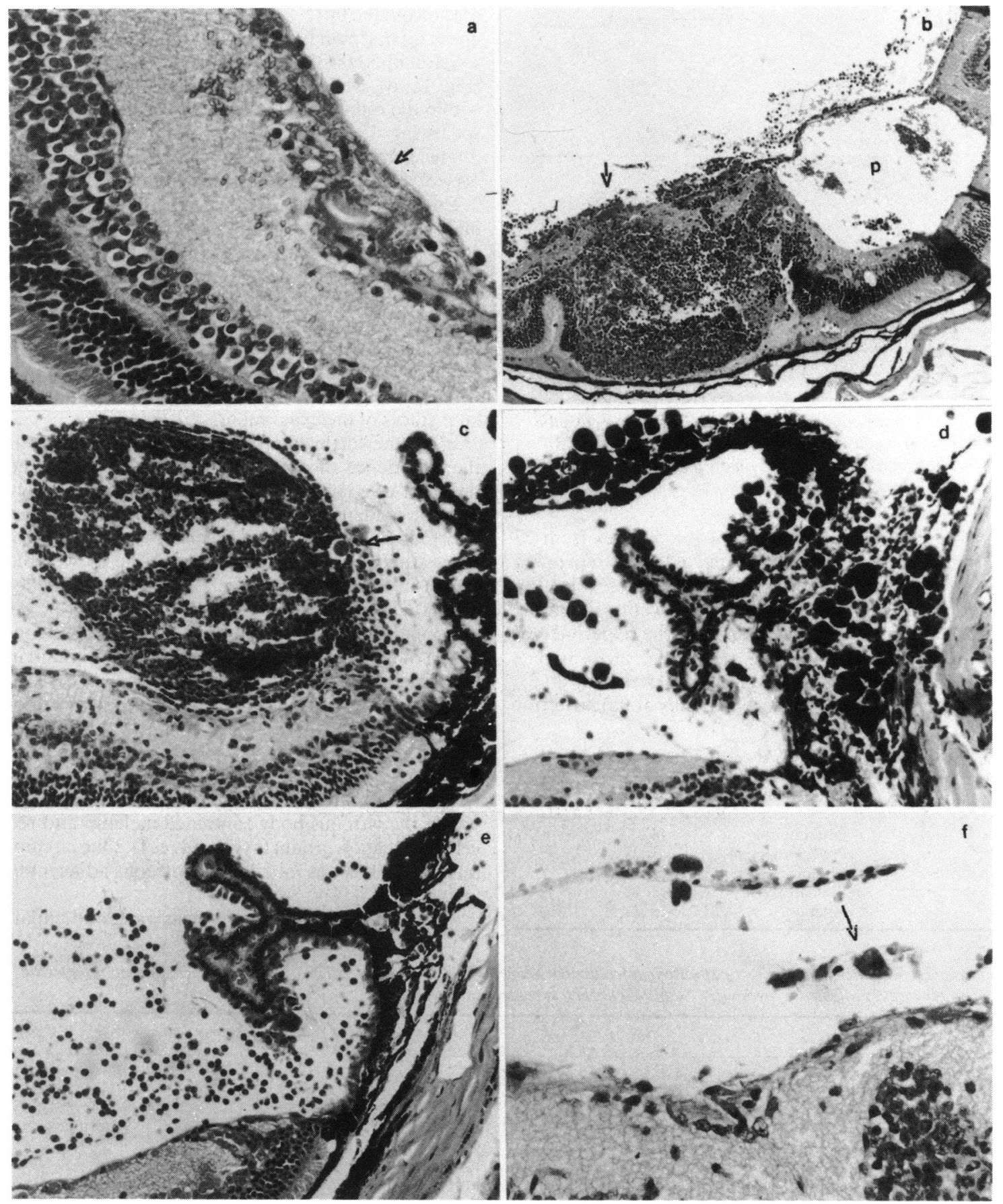

Fig. 3 Illustrations of the intraocular reactions (paraffin sections stained $H$ and $E$ ). a: Superficial microinfarct $(\uparrow)$ at day 6 $(\times 400)$. b: Haemorrhagic pseudocyst $(p)$ adjacent to a retinal granuloma $(\uparrow)(\times 160)$. c: A mixed inflammatory infiltrate with an intact larva $(\uparrow)$ in the peripheral vitreous $(\times 400)$. d: Marked iridocyclitis at day $32(\times 630)$. e: Low-grade iridocyclitis and pars planitis with vitreous infiltration at day $41(\times 400)$. $\mathrm{f}$ : Macrophages $(\uparrow)$, red cells, and lymphocytes in the vitreous $(\times 630)$. 


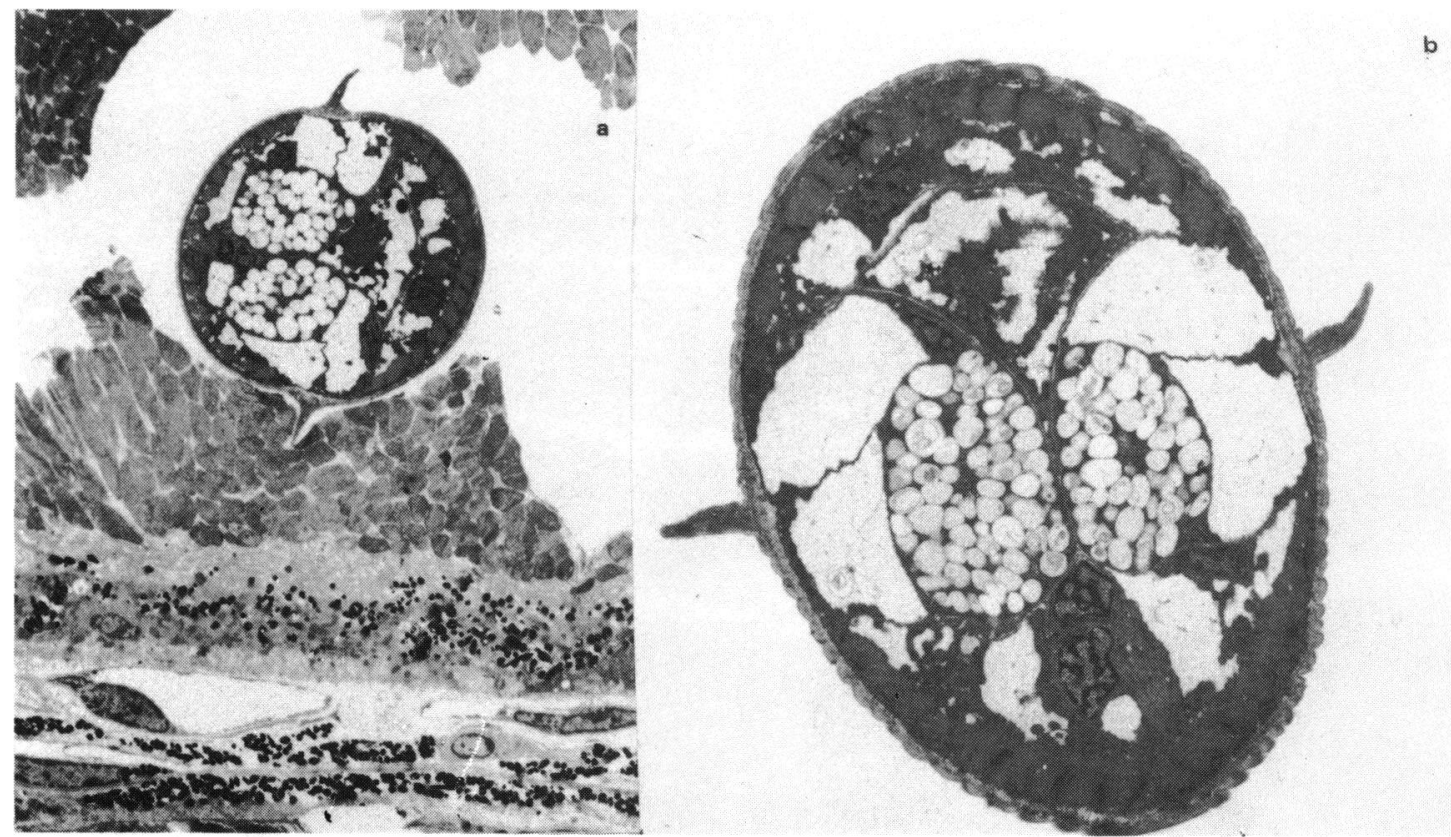

Fig. 4 Electron micrographs showing intact internal structures of Toxocara canis second-stage larva $(\times 2083)$.

from a solitary lesion in the orbit, occurred in the retina in 10 eyes which had been removed after the 6th day (Fig. 3b, 5). The granulomas consisted of clusters of epithelioid macrophages with small inconspicuous multinucleate giant cells (Fig. 6a). The clusters of macrophages were surrounded by a mantle of lymphocytes and polymorphonuclear leucocytes of neutrophil type. Eosinophil poly-

Retinal Granuloma Incidence

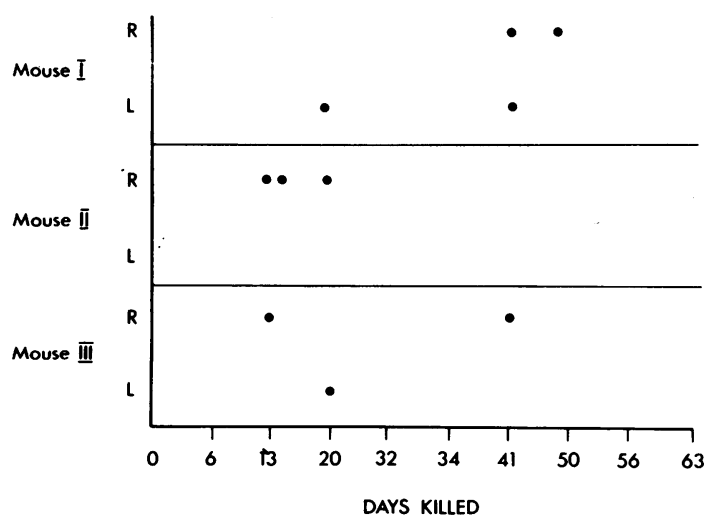

Fig. 5 Retinal granuloma incidence in eyes of infected mice. $R$ if the right eye, $L$ is the left eye. I, II, and III denote a set of 3 randomly chosen mice killed on the days noted in the horizontal axis. morphonuclear leucocytes were not observed at any stage of the inflammatory process. It was also remarkable that the choroid and choriocapillaris were free from inflammatory cell infiltration in all the specimens examined. Careful search in these granulomas failed except on one occasion (Fig. 3c) to demonstrate intact larvae or fragments of larvae. Optic nerve involvement (Fig. 6b) was relatively uncommon (Fig. 6a), and an orbital granuloma was found in only one specimen (Fig. 6c).

Additional features of interest included preretinal cellular infiltration (Fig. 6d) and the presence of isolated aggregates of macrophages within the photoreceptor layer (Fig. 6e, f).

\section{Discussion}

Murine ocular toxocariasis in the C57 black strain offered a good animal model for study. Previous work by Olsen' on Swiss Yale mice extended over 34 days but did not illustrate the histopathological changes described in the text. Studies on a guinea-pig model for ocular toxocariasis considered the role of IgE antibodies and mast cells in detail, ${ }^{10}$ but this species is atypical in its immune response. In addition this may not be pertinent to the human disease, since the guinea-pig does not play a part in the life cycle of the parasite, whereas the mouse can be a paratenic host and hence does transmit the disease when ingested by the dog. The present mouse model, 


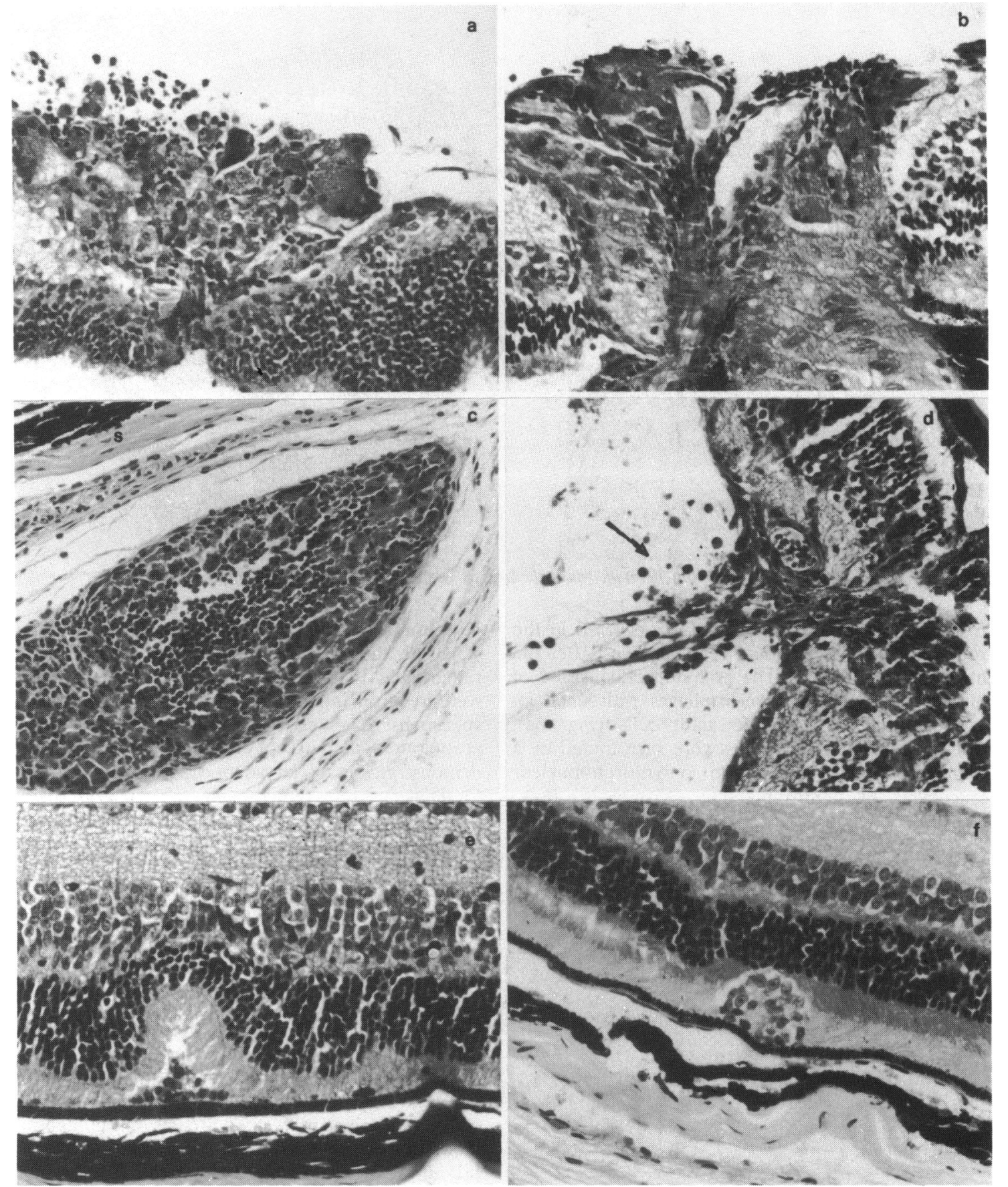

Fig. 6 Features of inflammatory process (paraffin sections stained $H$ and $E$ ). a: Giant-cell granulomatous reaction in the retina at day 41. b: Papillitis with vasculitis in central vessels at day $32(\times 630)$. c: A well circumscribed granulomatous reaction external to sclera $(S)$ at day $34(\times 630)$. d: Peripheral retina showing preretinal cellular proliferation $(\uparrow)(\times 630)$. $\mathrm{e}$ and $\mathrm{f}$ : Clusters of macrophages within the photoreceptor layers $(\times 750)(\times 630)$. 
studied over a more extended period, simulated the basic pathological changes described in human histological studies, namely, (a) diffuse nematode endophthalmitis, $(b)$ posterior pole granulomata, and $(c)$ peripheral retinitis. ${ }^{1311}$

At the cellular level the early responses at day 6 were predominantly those of a neutrophil polymorphonuclear leucocytic infiltration with vasculitis and retinal microinfarction. From day 13 onwards there was an increase in the macrophage component, and the distribution of these infiltrates suggested that the sites of entry were mainly the pars plana, the optic disc, and the retinal (but never the choroidal) blood vessels. Although macrophages were found within the outer segments in the subretinal space, and in general there was diffuse retinal infiltration, the choroidal stroma was notably free from infiltration.

Marked iridocyclitis and pars planitis were found. Hypopyon or iris nodules were not observed on external examination as has been reported in human cases. ${ }^{12}$

Granuloma formation was noted between days 13 and 50 and occurred mainly at the posterior pole. Although on 2 occasions larvae and granulomas were seen at separate locations within the same eye, on only 2 occasions was a larva or the remnants of a larva seen within a granuloma in the many serial sections which were examined. The granulomatous reaction consisted of macrophages, multinucleate giant cells, lymphocytes, and plasma cells, but eosinophils were inconspicuous even with the application of a special stain. The possibility of degranulation of eosinophils cannot, however, be excluded as an explanation for their apparent absence from the tissue. It is relevant to add that, in the myotropic phases of murine toxocariasis, eosinophils are present in the early poorly defined granulomas but are rapidly replaced by epithelioid macrophages in the mature granuloma in the third week of infection. ${ }^{13}$ By contrast in the neural tissue in the present model ${ }^{14}$ and unpublished data (Smith) there is little or no response around migrating second-stage larvae or their migratory pathways. Thus the inflammatory changes seen in the ocular disease may represent an intermediary response in the myotropic and neurotropic phases due to the unique character of the formative tissues of the eye.

The route of entry of larvae into the eye is vascular, and in this study larvae were demonstrated in retinal capillaries. None was found in the choroidal vessels. This is pertinent to the clinical observation ${ }^{15}$ which has shown that larvae can enter the eye via the retinal blood vessels before entry into the vitreous. Perilimbal infiltrates (composed of polymorphonuclear leucocyte cells) were noted, but larvae were not found in the cornea or anterior segment, though their presence has been reported clinically. ${ }^{3}$
A consistent finding in this study was that larvae or larval remnants were not seen within the centre of a granuloma. The presence of a larva or larval remnants within a necrotising granuloma containing eosinophils is, however, a diagnostic pathological feature of human toxocariasis. ${ }^{1}$ The apparent absence of larval forms from the ocular granulomas observed in this model implies that the larvae do not have to be present to propagate the mature granuloma which may have been stimulated initially by them. This suggestion is supported by the observation that larvae are not seen in early granulomas in murine visceral larva migrans, and Kayes and Oakes ${ }^{13}$ have suggested that Toxocara second-stage larvae can migrate out of an early granuloma.

Toxocara sp. second-stage larvae are rapid tissue migrators which have individual tracts in the tissues through which they migrate. Recent work has shown that the larvae have a capacity for rapid metabolic turnover of their outer antigenic glycosylated surface proteins. ${ }^{16}$ This turnover is complete within 3 hours, and antibodies raised against the excretions/secretions are unable to bind to the outer larval surface at body temperature. This rapid surface turnover may account for the inability of antibody-producing or cytotoxic immune cells, individually or in combination, to attach to the surfaces. By this evasive mechanism the parasite may avoid larval entrapment within a granuloma. Thus, although the larvae or their products may incite granuloma formation, their continued presence may not be necessary for the propagation of the immune response. The hypothesis that by rapid migration ${ }^{17}$ and surface protein turnover larvae escape entrapment would account for the fact that inflammatory cells were inconspicuous in the surrounding tissues when larvae were observed in the retina or retinal vessels. Another possible explanation for the latter observations is that larvae can absorb host surface antigens on to their surfaces or produce parasite-specific antigens which cross-react with blood group antigens, ${ }^{18}$ thus providing them with an antigenic disguise. ${ }^{19}$

We thank James Ralston and Dorothy Aitken for their valuable technical help, Jennifer Murray for typing the manuscript, John Hendry for the photographs, and the Medical Illustrations Department, Western Infirmary, for the diagrams.

This work was supported in part (H.V.S. and R.W.A.G.) by MRC grant no. G8118073/T.

\section{References}

1 Duguid IM. Features of ocular infestation by Toxocara. Br J Ophthalmol 1961; 45: 789-96.

2 Dean-Hart JC, Raistrick ER. Adult toxocariasis. Trans Ophthalmol Soc UK 1977; 97: 164-7.

3 Brown DH. Ocular Toxocara canis. J Pediatr Ophthalmol 1970; 7: $182-92$.

4 Duguid IM. Chronic endophthalmitis due to Toxocara. Br J Ophthalmol 1961; 45: 705-17. 
5 Wilder HC. Nematode endophthalmitis. Trans Am Acad Ophthalmol Otolaryngol 1950; 55: 99-109.

6 Nichols RL. The aetiology of visceral larval migrans. Diagnostic morphology of infective second stage toxocara larvae. J Parasitol 1956; 42: 349-62.

7 Perkins ES. Patterns of uveitis in children. Br J Ophthalmol 1966; 50: $169-85$.

8 Belmont JB, Irvine A, Benson W, O'Connor R. Vitrectomy in ocular toxocariasis. Arch Ophthalmol 1982; 100: 1912-5.

9 Olson LJ. Ocular toxocariasis in mice. Int J Parasitol 1976; 6: 247-51.

10 Rockey JH, Donnelly JJ, Stromberg BE. Immunopathology of ascarid infection of the eye. Arch Ophthalmol 1981; 99: $1831-40$.

11 Wilkinson CP, Welch RB. Intraocular Toxocara. Am J Ophthalmol 1971; 71: 921-30.

12 Liesegang TJ. Atypical ocular toxocariasis. J Pediatr Ophthalmol $1971 ; 14: 349-53$.
13 Kayes SG, Oakes J. Development of the granulomatous response in murine toxocariasis. Am J Pathol 1978; 93: 277-94.

14 Abo-Shehada. Studies of some factors affecting the migration and survival of larval Toxocara canis within two strains of mice. PhD thesis. University of Wales: 1982.

15 Byers B, Kimura SJ. Uveitis after death of a larva in the vitreous cavity. Am J Ophthalmol 1974; 77: 63-6.

16 Smith HV, Quinn R, Kusel JR, Girdwood RWA. Effects of temperature and antimetabolites on antibody binding to outer surface of second stage Toxocara canis larvae. Mol Biochem Parasitol 1981; 4: 183-93.

17 Croll NA, Mathews BE. Biology of nematodes. Glasgow: Blackie, 1977: 79.

18 Huntley CC, Lyerly A, Patterson MV. Isohemaglutinins in parasitic infections. JAMA 1969; 208: 1145-8.

19 Ogilvie BM, Worms MJ. Immunology of nematode parasites. In: Cohen S, Sadun EH. eds. Immunology of parasitic infections. Oxford: Blackwell Scientific Publications, 1976: 380-3. 